\title{
CONTACT-IMPACT SIMULATIONS ON MASSIVELY PARALLEL SIMD
}

SUPERCOMPUTERS

A.NL/CP -76686

DE93 004188

Edward J. Plaskacz

Argonne National Laboratory

Argonne, IL 60439-4845

U.S.A.

Ted Belytschko and Huai-Yang Chiang

Department of Civil and Mechanical Engineering

Robert R. McCormick School of Engineering and Applied Science

The Technological Institute

Northwestern University

Evanston, IL 60208-3109

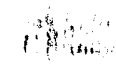

U.S.A.

ABSTRACT

The implementation of explicit finite element methods with contact-impact on massively parallel SIMD computers is described. The basic parallel finite element algorithm employs an exchange process which minimizes interprocessor communication at the expense of redundant computations and storage. The contact-impact algorithm is based on the pinball method in which compatibility is enforced by preventing interpenetration on spheres embedded in elements adjacent to surfaces. The enhancements to the pinball algorithm include a parallel assembled surface normal algorithm and a parallel detection of interpenetrating pairs. Some timings with and without contact-impact are given. 


\section{INTRODUCTION}

Structural vulnerability applications are highly compute intensive. In studies of crashworthiness, impact, and penetration, it is not unusual for an analysis to require 100 hours of CPU time on current generation production supercomputers, despite the relative simplicity of the models being studied. The difficulty in obtaining blocks of time this large in a production environment severely impairs the number of design options that can be investigated. The lack of software capable of simultaneously accurately capturing the physics of a crash event and exploiting the power of high-performance computer architectures necessitates costly experimental testing for design verification and certification. For example, in the design of automobiles for crashworthiness, hundreds of sled tests and dozens of full-vehicle crash tests are conducted for each new vehicle program. A sled test may cost $\$ 5,000$ and full-scale prototypes may cost as much as $\$ 750,000$ each. The cost can be much greater (on the order of $\$ 10 \mathrm{M}$ ) if a redesign results in retooling for a structural component late in the program. Attention has focused on highperformance computer architectures as an effective avenue to bridge the gap between computational needs and the power of computational hardware. New high-performance computer architectures promise order-of-magnitude increases in computational performance, thereby allowing the numerical laboratory to replace physical experiments to a much greater degree.

Order-of-magnitude increases in the computational speed over a conventional vector supercomputer have been attained in research conducted at Argonne for three-dimensional nonlinear analysis of homogeneous (identical element type and material model) shell structures on single-instruction multiple-data stream (SIMD) architectures. The adaptation of a finite element program with explicit time integration and contact-impact to a massively parallel SIMD computer, the Connection Machine, holds the promise of bringing the power 
of this novel architecture to bear on compute intensive applications such as crashworthiness and armor penetration while still retaining these performance gains.

The Connection Machine (CM2) system consists of a front end computer, such as a SUN workstation, and a parallel processing unit consisting of up to $64 \mathrm{~K}$ data processors, each with local memory, and (optionally) I/O systems that support mass storage and graphics display devices. Processors are connected in a hypercube topology. With 16 processors per vertex, a 12-dimensional hypercube with 4096 vertices can arrange 65,536 $(64 \mathrm{~K})$ processors with no processor more than 12 wires away.

The locality of data storage becomes a severe impediment in contact-impact simulations. To simulate impact of two bodies modelled by finite element meshes, it is necessary to check whether the elements of one mesh interpenetrate the other mesh. On a massively parallel computer, a complete check over all possible master-slave element pairs for possible interactions would saturate the system with interprocessor communication. A parallel pinball algorithm which reduces the set of elements to be checked for interpenetration is discussed.

The outline of this paper is as follows. In the next section, the finite element algorithm which has been developed for explicit methods on massively parallel computers with local memory will be described. Section 3 describes the pinball contact-impact algorithm. Section 4 describes the Connection Machine algorithm for a two-body contactimpact algorithm. Some results and timings on the CM are given in Section 5.

\section{FINITE ELEMENT ANALYSIS ON THE CONNECTION MACHINE}

A four-node isoparametric quadrilateral element for 2D transient, nonlinear solid mechanics was implemented to design the algorithm and benchmark the performance of the Connection Machine. The governing equations for a finite element semidiscretization by a Lagrangian mesh are: 
Momentum equation

$$
\begin{aligned}
\operatorname{Miv}(t) & =f(t) \\
f(t) & =f_{\text {ext }}(t)-f_{\text {int }}(t) \\
f_{\text {ext }}(t) & =\sum_{e} L_{e}^{T} f_{\text {ext }}^{e}(t) \\
f_{\text {int }}(t) & =\sum_{e} L_{e}^{T} f_{\text {int }}^{e}(t) \\
f_{\text {int }}^{e}(t) & =\int_{\Omega} B^{T}(t) \sigma(t) d \Omega
\end{aligned}
$$

Measure of deformation

$$
\begin{aligned}
\dot{\varepsilon}(t) & =B(t) v_{e^{(t)}} \\
v_{e}(t) & =L_{e} v(t)
\end{aligned}
$$

Constitutive equation

$$
\stackrel{\nabla}{\sigma}(\mathrm{t})=\text { function }(\dot{\varepsilon}(\mathrm{t}), \sigma)
$$

In the above

$$
\begin{aligned}
\mathbf{M} & =\text { mass matrix; } \\
\mathbf{v}(t) & =\text { nodal velocity matrix }
\end{aligned}
$$

$f(t), f_{\text {ext }}(t), f_{\text {int }}(t)=$ resultant, external and internal nodal forces, respectively ;

$\mathbf{B}(t)=$ semidiscrete form of the symmetric part of the gradient operator;

$\dot{\varepsilon}(t)=$ rate-of-deformation

(velocity strain) tensor; 


$$
\begin{aligned}
\sigma(t) & =\text { Cauchy stress tensor; } \\
\mathbf{L}_{\mathrm{e}} & =\text { Boolean connectivity matrix of element } \mathrm{e} .
\end{aligned}
$$

The implementation of the direct vector method for explicit time integration $1,2,3$ required a substantial redesign of the Von Neumann algorithm and underlying data structure for use on the Connection Machine. Since the computational capabilities of the Connection Machine far exceed its communication abilities, careful attention must be devoted to efficient interprocessor communication. An understanding of this procedure and its data structure is necessary to explain its extension to contact-impact simulations.

First, the amount of interprocessor communication was minimized. The Exchange algorithm $4,5,6$ was developed which minimizes interprocessor communication at the expense of redundant computations and storage. The data structure combined the material and geometric variables of the element with the kinematic, mass, and force variables of the adjacent nodes. At each time step only internal forces are exchanged between processors. No kinematic variables are exchanged, instead the equations of motion are integrated for all of the nodes of the element, even though this involves redundant computation. The decrease in communication time more than compensates for the additional calculations. A schematic diagram comparing the data structure of the SISD (Von Neumann) and SIMD $(\mathrm{CM})$ is shown in Figure 1. The differences in the associated algorithms are highlighted in Tables 1 and 2 . 


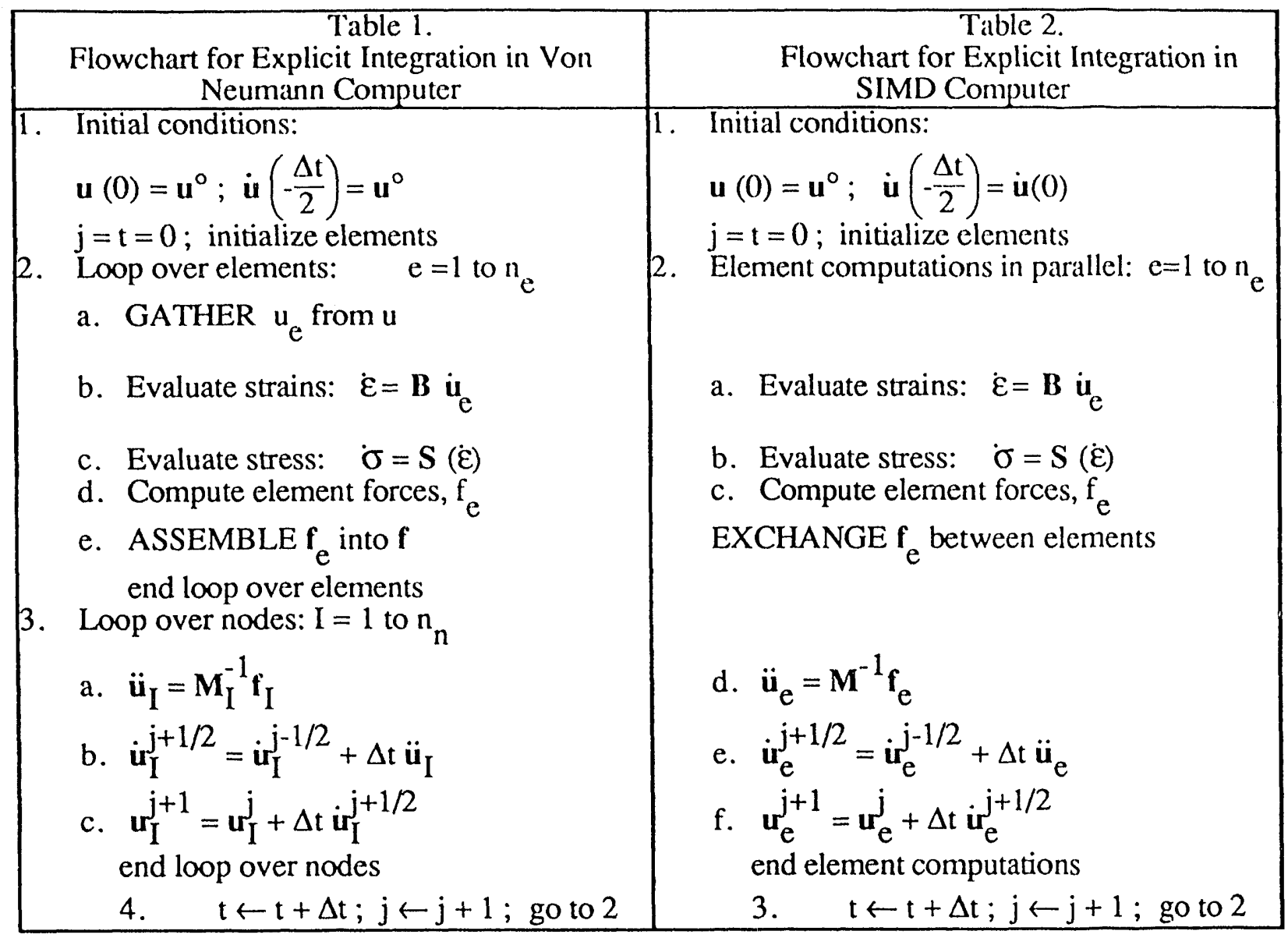

Second, the mode of interprocessor communication must be regulated. Router interprocessor communication allows an exchange of data between any two processors of the Connection Machine. Nearest neighbor (NEWS) interprocessor communication is an order of magnitude faster than general (Router) communication. NEWS requires a structured mesh, i.e. a mesh where adjacent elements can be mapped to adjacent processors. The four-stage internal force exchange with NEWS is diagrammed in Figure 2 .

Most large finite element meshes may be decomposed into subdomains which are highiy structured. Thus a hybrid NEWS-Router communication scheme ${ }^{5,6}$, where NEWS is used for exchange of internal forces within structured subdomains and the Router is used to exchange nodal forces between subdomains, can be used to exploit this partial mesh 
structure. A schematic depiction of the hybrid NEWS-Router interprocessor communication scheme is depicted in Figure 3.

The key to effective programming of a SIMD computer is the choice of an appropriate data structure which is duplicated across the local processor memories and operated on concurrently by each processor. The $\mathrm{C}$ programming language allows a set of related variables to be grouped together by declaring them to be members of a structure. A structure can be viewed as a generalization of an array. Whereas the variables of an array are restricted to one data type, the variables within a structure can be of any data type even other structures. In $\mathrm{C}^{*}$, a structure that defines the memory layout of a processor is called a domain. $\mathrm{C}^{*}$ extends the concept of a structure by allowing functions to be associated members of the domain. The $\mathrm{C}^{*}$ code is nearly identical to the $\mathrm{C}$ code which would be written for a single element problem.

To program the Exchange algorithm in CM FORTRAN required recasting the finite element data structure into parallel multidimensional arrays with complex interrelationships. CM FORTRAN is an implementation of Fortran 77 supplemented with the arrayprocessing extensions from the ANSI standard Fortran 90. Other Fortran 90 features such as pointers, structures, modules, and precision control - are currently not part of CM Fortran. The ability, present in $\mathrm{C}^{*}$, to group various data types and form a parallel object which is replicated across all of the local processor memories is not available in CM FORTRAN.

The underlying data model in CM FORTRAN is an array. CM FORTRAN was built with the idea of a distribution of work between the two components of a CM system, i.e. the front end and parallel processing unit. The home of a CM FORTRAN array may be either the front end or the parallel processing unit. Arrays are allocated by how they are used. Arrays that are used only in FORTRAN 77 constructs are stored and processed on the front end. Arrays that are used in FORTRAN 90 constructs are stored and processed 
on the parallel processing unit. Alternatively, the CM FORTRAN programmer can specify where the array is stored by a LAYOUT directive.

In the absence of compiler directives, $\mathrm{CM}$ arrays are laid out one array element per processor. The LAYOUT directive provides a mechanism by which this canonical layout may be altered. Multidimensional arrays may be created with parallel and serial dimensions. For example, a three dimensional array with one parallel dimension and two serial dimensions may be viewed as a set of local matrices.

Data movement in CM FORTRAN is less transparent to the programmer. While the underlying mechanisms (Router and NEWS) are the same, the CM FORTRAN compiler makes the decision as to which mechanism or combination of mechanisms is most appropriate. Vector-valued subscripts, where an integer vector serves as a sequence of index values in an array reference, always use the router communication network. CM FORTRAN provides an intrinsic functions analogous to NEWS for moving array elements in regular patterns along array dimensions (CSHIFT). The CM FORTRAN compiler maps a CSHIFT instruction directly onto a NEWS instruction.

The following definitions illustrate the data structure for a mesh of two dimensional quadrilaterals into CM FORTRAN parallel arrays:

$\begin{array}{ll}\text { REAL, ARRAY(1:NPE, 1:MAX_ROWS, 1:MAX_COLS) } & :: \text { X } \\ \text { REAL, ARRAY(1:NPE, 1:MAX_ROWS, 1:MAX_COLS) } & :: \text { Y } \\ \text { REAL, ARRAY(1:MAX_ROWS, 1:MAX_COLS) } & :: \text { AREA } \\ \text { REAL, ARRAY(1:MAX_ROWS, 1:MAX_COLS) } & :: \text { THICK } \\ \text { REAL, ARRAY(1:MAX_ROWS, 1:MAX_COLS) } & :: \text { DENSTY } \\ \text { REAL, ARRAY(1:NPE, 1:NDGR, 1:MAX_ROWS, 1:MAX_COLS) } & :: \text { MASS } \\ \text { REAL, ARRAY(1:MAX_ROWS, 1:MAX_COLS) } & :: \text { YM } \\ \text { REAL, ARRAY(1:NPE, 1:NDGR, 1:MAX_ROWS, 1:MAX_COLS) } & :: \text { ACC } \\ \text { REAL, ARRAY(1:NPE, 1:NDGR, 1:MAX_ROWS, 1:MAX_COLS) } & :: \text { VEL } \\ \text { REAL, ARRAY(1:NPE, 1:NDGR, 1:MAX_ROWS, 1:MAX_COLS) } & :: \text { DISP }\end{array}$


$\begin{array}{lc}\text { REAL, ARRAY(1:NPE, 1:NDGR, 1:MAX_ROWS, 1:MAX_COLS) } & :: \text { FINT } \\ \text { REAL, ARRAY(1:NPE, 1:NDGR, 1:MAX_ROWS, 1:MAX_COLS) } & :: \text { FEXT }\end{array}$

In the above:

MAX_ROWS is the number of element rows in the mesh

MAX_COLS is the number of element columns in the mesh

NPE is the number of nodes per element

NDGR is the number of degrees of freedom per node

Layout directives are necessary to inform the compiler of the mapping between Connection Machine processors and array elements.

CMF\$ layout X(:serial, :news, :news)

CMF\$ layout Y(:serial, :news, :news)

CMF\$ layout AREA(:news, :news)

CMF\$ layout THICK(news, :news)

CMF\$ layout DENSTY(:news, :news)

CMF\$ layout MASS(:serial, :serial, :news, :news)

CMF\$ layout YM(:news, :news)

CMF\$ layout ACC(:serial, :serial, :news, :news)

CMF\$ layout VEL(:serial, :serial, :news, :news)

CMF\$ layout DISP(:serial, :serial, :news, :news)

CMF\$ layout FINT(:serial, :serial, :news, :news)

CMF\$ layout FEXT(:serial, :serial, :news, :news)

In the above, ACC is the acceleration, VEL is the velocity, DISP is the displacement, FINT is the internal force, and FEXT is the external force. For a twodimensional quadrilateral, each element would have four nodes, each node would in turn have two degrees of freedom. Therefore, each of these four-dimensional arrays (ACC, VEL, DISP, FINT, and FEXT) is a set of $4 \times 2$ matrices stored in each processor. 


\section{PINBALL ALGORITHM}

Implementation of the assembled surface normal 7,8 and pinball ${ }^{9}$ algorithms, greatly reduces the time required for the detection of sliding interfaces. In principle, to simulate impact of two bodies modeled by finite element meshes, it is necessary to determine the interpenetrating groups of elements. The search space for interpenetrating elements can be restricted by immediate elimination of all elements whose nodes are all interior to the mesh. Those elements cannot interpenetrate the elements of another body. Secondly, the pinball algorithm replaces the complex interpenetrability checks between two hexahedron or quadrilaterals with a check of the distance between two spheres.

These two algorithms are shown in Figure 4. First, the assembled surface normal algorithm is employed to determine which elements are adjacent to the surface. An element is on the surface of the mesh if it has at least one node with a non-zero surface normal; otherwise it is an interior element and need not be checked for interpenetration. Second, spheres or pinballs are embedded in the surface elements. The detection of impacting pairs becomes, computationally, a very simple procedure. The distance between the centers of each slave pinball and each master pinball is then calculated and compared with the sum of the radii of the two elements.

A penalty force proportional to the penetration depth is calculated and applied to the nodes of the two interpenetrating elements. The penalty forces, along with the forces arising from element stresses and externally applied loads, are used in the calculation of the nodal accelerations.

The center and radius of the pinball embedded in element e are given by:

$$
\begin{aligned}
& c_{i c}=\frac{1}{4} \sum_{I=1}^{4} x_{i}^{c} I \\
& R=\sqrt{\frac{A^{e}}{\pi}}
\end{aligned}
$$


respectively, where $c_{i}$ are the coordinates of the center of the pinball, $x_{i I}^{c}$ are the coordinates of node I of element $\mathrm{e}, \mathrm{R}$ is radius of the pinball, and $\mathrm{A}^{\mathrm{c}}$ is the area of element e.

The center of each pinball is simply the average of its nodal coordinates while the radius is determined by setting the area of the pinball equal to the area of the element itself. For elastic-plastic problems most of the element deformation can be considered nearly incompressible; therefore, the element area, and also the radii of the pinballs, will change little over the course of the simulation. For this reason, we calculate the radii only once and consider them to be constant thereafter. The coordinates of the pinballs, however are calculated every time step. For materials with substantial compressibility this assumption of nearly constant area would be incorrect and the radius for each element would have to be recalculated every few steps.

The detection of the impacting pairs is, computationally, a very simple procedure. The distance between the centers of each slave pinball and each master pinball is calculated and then compared with the sum of the radii of the two elements. Interpenetration has occurred when

$$
d_{i j}<R_{i}+R_{j}
$$

where $d_{i j}$ is the distance between the centers of elements 1 and 2 and $R_{i}, R_{j}$ are the radii of elements $\mathrm{i}$ and $\mathrm{j}$. Note that each pinball may be penetrated by more than one pinball during a time step.

A penalty method is used for contact-impact. The penalty force is proportional to the depth of penetration between the two elements; therefore the next step of the procedure is to determine the penetration depth of the two elements. In the pinball algorithm, the penetration depth $g$ is easily calculated. Consider two interpenetrating pinballs, 1 and 2 
with the velocities $\mathbf{v}_{1}$ and $\mathbf{v}_{2}$; the normal of the associated surfaces are $\mathbf{n}_{1}$ and $\mathbf{n}_{2}$. The position vectors of the centers of the two pinballs are given by $\mathbf{c}_{1}$ and $\mathbf{c}_{2}$. The penetration is given by $g$ and is defined as the relative displacement of the centers of the pinballs in the average normal direction, so that the following equation determines $g$

$$
\left\|c_{1}-c_{2}+g n\right\|^{2}=\left(R_{1}+R_{2}\right)^{2}
$$

and the average normal $\mathbf{n}$ is given by

$$
\mathbf{n}=\frac{1}{2}\left(\mathbf{n}_{2}-\mathbf{n}_{1}\right)
$$

This gives

$$
g=-b+\sqrt{b^{2}-c}
$$

where

$$
\begin{aligned}
& b=\frac{n_{i}\left(x_{i}-c_{i 2}\right)}{n_{j} n_{j}} \\
& c=\frac{x_{i} x_{i}+c_{i 2} c_{i 2}-2 x_{i} c_{i 2}-\left(R_{1}+R_{2}\right)^{2}}{n_{j} n_{j}}
\end{aligned}
$$

Note that only the positive sign on the radical in (14) need be considered; the negative root corresponds to a negative value of $g$ which is irrelevant.

The penalty force which will be applied to the nodes of each element is proportional to the penetration depth and is given by

$$
\mathbf{f}^{\mathbf{p}}=\left(\mathrm{p}_{1} \dot{\mathrm{g}}+\mathrm{p}_{2} \mathrm{~g}\right) \mathbf{n}
$$


where $\mathbf{n}$ is the average normal given by (13). An expression for the parameter, $p_{2}$, has been proposed for the case of a node impacting the surface of an element and the volume, area and bulk modulus referred to the impacted element

$$
p_{2}=\frac{\beta B A^{2}}{V}
$$

where B, A, and V are the bulk modulus, area of the impacted surface, and volume of the element, $\mathbf{f}^{\mathrm{P}}$ is the penalty force on the pinball. In the present context, the properties of two pinballs must be ccnsidered, so the penalty parameter will be given by

$$
p_{2}=\frac{1}{2} \beta\left(B_{1} R_{1}+B_{2} R_{2}\right)
$$

where $B_{1}, B_{2}$ are the bulk moduli of the impacting pinballs, and $R_{1}, R_{2}$ are the radii of the impacting pinballs. Equation (15) gives the contact force that will be applied in opposite directions to each of the two impacting pinballs. This force is then divided among the four nodes of each element.

$$
\mathbf{f}_{N}^{\mathrm{P}}=\frac{1}{4} \mathbf{f}^{\mathrm{P}} \quad \mathrm{n}=1,4
$$

where $f_{N}^{\mathrm{PP}}$ are the element level penalty force on local node $\mathrm{n}$ of the element. These forces are then assembled to the global force vector as usual.

The penalty force is divided among the four nodes of the quadrilateral to preserve the symmetry of the underlying linearized system. Since the position of the pinball depends on the four nodes of the quadrilateral, the linearized equations would not be symmetric if the force were subdivided only among the surface nodes; an alternative 
algorithm where $\mathrm{c}$ depends only on the surface nodc velocities and hence the penalty forces are distributed only to the surface nodes is now under investigation.

The penalty forces, along with the forces arising from element stresses and externally applied loads, are used in the calculation of the nodal accelerations. Therefore the contact routine appears in the algorithm immediately before the nodal accelerations are calculated.

PARALLEL IMPACT-CONTACT SIMULATIONS ON THE CONNECTION MACHINE

\section{Mapping Strategy}

A contact-impact simulation between two bodies and the mapping between the finite elements in the two coarse meshes and Connection Machine processors is shown in Figure 6. An example of a two-body problem is the penetration of a projectile into a target. The Connection machine processors are partitioned into two sections, one section for each impacting body. The partition can be arbitrary if a boolean array, BNDRY, is constructed ( 1 for element sides on the interior of each mesh and 0 for element sides on the mesh boundaries) to describe the allocation of Connection Machine processors between the two bodies. The processors perform identical computations throughout most of the simulation. NEWS interprocessor communication is used to exchange internal forces among elements in the penetrant (siave) and target (master) meshes simultaneously. The BNDRY array prevents an unintentional exchange of internal forces between the two bodies. 
Parallel Assembled Surface Normal Algorithm

In production automobile crashworthiness and armor penetration problems, the total number of elements in the simulation is on the order of $10^{3}$ to $10^{4}$. A parallel SIMD assembled surface normal algorithm has been introduced here to restrict the possible contact areas. The resulting surface normals are shown in Figure 7 . These normals are then exchanged in a manner identical to the four stage exchange of element internal forces shown in Figure 2. The resultant element surface normals are shown in Figure 8, with pinballs injected into the elements with non-zero surface normals.

\section{Parallel Pinball Algorithm}

The pinball algorithm has proved to be highly vectorizable. However, it is still a challenge to implement it effectively on a modern massively parallel computer. The parallel extension we propose here takes advantage of the massive storage capacity of this kind of computer to accelerate the search for contacting pairs. The procedure is summarized in Table 3. After the surface elements have been detected by the parallel assembled surface normal algorithm, the data necessary to detect contacting pairs and compute penalty forces, i.e. the radius, pinball centroid coordinates, and bulk modulus, is collected for both the master and slave surface elements. The slave element information is then distributed to each master element and the master element information is distributed to the slave elements. The search for contacting pairs and computation of penalty forces can then proceed in parallel. Although the collection and distribution of this data for each time step entails communication overhead, it is more than offset by parallel searching.

The PACK and UNPACK intrinsic functions can be applied to collect and distribute the data. The transformational function PACK packs into a rank-one array (vector) those elements of an array that are selected by a logical array of conforming shape, and the transformational function UNPACK performs the reverse operation. The following CM FORTRAN statements provide some of the details behind the collection and distribution of slave data: 
First, a WHERE statement is used to test the element surface normal value in the slave processors. Slave processors whose element surface normal is non-zero in magnitude have a value of 1 assigned to their ISLVPB array element.

WHERE ((ABS(XENORM) .GT. 0.0 .OR. ABS(YENORM) .GT. 0.0)

.AND. (ISLV .EQ. 1)) ISLVPB = 1

Next, pinballs are injected into those slave processors whose ISLVPB array element is equal to 1 . The pinball centroid coordinates are stored in the arrays XCEN and YCEN. Finally the expression, ISLVPB .EQ. 1, is used as a mask to filter out all of the slave elements without pinballs. XSS and YSS are packed arrays of slave pinball coordinate data.

$$
\begin{aligned}
& X S S=P A C K(X C E N, \text { MASK }=\text { ISLVPB } . E Q .1) \\
& Y S S=\text { PACK }(Y C E N, \text { MASK }=\text { ISLVPB } . E Q .1)
\end{aligned}
$$

The XSS and YSS are sent to all master elements. Then, similar steps are performed to inject pinballs and compute the coordinates of their centroids for the processors assigned to the master elements. 
Table 3 Flowchart for the Parallel Pinball Algorithm

1. For all surface elements, PACK the data necessary for the detection of contact and computation of penalty forces.

2. Pass the slave element data to the master elements and vice versa.

3. Perform a parallel search and penalty force computation for interpenetrating pairs. In each processor,

a. Compare distance between centroid and each surface element centroid from the other body to the sum of their radii.

b. Calculate penalty forces for all interpenetrating pairs.

4. Use UNPACK to convert the penalty force vector into a $2 \mathrm{D}$ array.

5. Get the total penalty forces for each element by summing the contributions from each contacting element in the other domain.

\section{RESULTS}

First, we report the results for the non-contact cases. A two-dimensional elastic wave propagation problem was run on a $16 \mathrm{~K}$ processor Connection Machine using two structured meshes, one with 8192 elements, the second with 16384 elements. In these structured meshes, the exchange process is accomplished with NEWS or nearest neighbor communication. The run times for these meshes are reported in Table 4 for both $\mathrm{C}^{*}$ and CM FORTRAN versions. It is readily observed that the CM FORTRAN version is about twice as fast. Furthermore, these results may be extrapolated to approximate the run time of a $64 \mathrm{~K}$ element mesh on a $64 \mathrm{~K}$ processor machine. The run time of a $16 \mathrm{~K}$ element mesh on a $16 \mathrm{~K}$ processor machine will be approximately equal to the run time of a $64 \mathrm{~K}$ element mesh on a $64 \mathrm{~K}$ processor machine. Therefore, the element cycle, i.e. the time required to 
update one element for a single time step, would be $0.56 \times 10^{-6} \mathrm{sec}$ for the $64 \mathrm{~K}$ element mesh on the $64 \mathrm{~K}$ processor machine. The element cycle is computed by dividing the total wall clock time by the product of the number of elements and the number of time steps. This element cycle time for the Connection Machine is approximately 16 times faster than that observed on a vectorized version of the program on the CRAY X-MP.

Table 4

2D Elastic Wave Propagation Problem With 4 Node Quadrilaterals; 7500 time steps; wall-clock times in seconds

\begin{tabular}{|c|c|c|}
\hline Number of Elements & C version & FORTRAN version \\
\hline \hline 8192 & 563. & 286. \\
\hline 16384 & 652. & 321. \\
\hline
\end{tabular}

We then tested the parallel algorithm against a serial search for interacting element pairs. A total of $16 \mathrm{~K}$ elements were used with the penetrant mesh consisting of $8 \mathrm{~K}$ elements and the target mesh consisting of $8 \mathrm{~K}$ elements. The simulation was run for 100 time steps. The parallel assembled surface normal algorithm identified the 2000 surface elements between the two bodies. A serial search for interacting element pairs required 631 seconds while the parallel algorithm described above required 67 seconds. The parallel algorithm results in nearly an order-ofmagnitude speed up over the sequential algorithm, despite the additional communication overhead. Nevertheless, the detection of interacting pairs remains the most computationally demanding part of the simulation.

\section{DISCUSSION AND CONCLUSION}

Proponents of the SIMD or data-parallel programming paradigm emphasize it is a natural approach for a data-intensive applications. The success of a SIMD architecture 
relies heavily on the homogeneity of the underlying data - homogeneous data leads to homogeneous computations which in turn leads to strong SIMD performance. Production finite element meshes are seldom totally homogeneous. Proponents of the MIMD programming paradigm are quick to point out that for inhomogeneous problems with many different types of elements and materials, SIMD machines like the Connection Machine 2 experience a drop in performance. For example, if a $64 \mathrm{~K}$ mesh consisting of five types of elements were run on a $64 \mathrm{~K}$ machine, only $20 \%$ of the speed on a uniform mesh could be achieved, since the five types of elements would have to be processed sequentially. For most engineering simulations, the catagories of inhomogeneities (i.e. number of elements and number of material types) are small relative to the number of processors in a massively parallel machine. Therefore, some combination of SIMD and MIMD would be ideal. Future entries in the high-performance supercomputer arena promise friendly environments where SIMD is supported by data parallel languages and MIMD is supported by message passing.

Bringing the power of the Connection Machine 2 to bear on two-body contactimpact simulations required dividing the processors into two blocks; each dedicated to one body. Since the identities of contacting elements are not known apriori, an interpenetration check is necessary. In the absence of shared memory, this check is difficult to implement. Careful attention must be paid to algorithm restructuring to control the amount of communication overhead. In this paper, a SIMD parallel assembled surface normal algorithm has been presented which uses the same communication paths as the exchange of internal forces to identify surface elements. Data necessary for the detection of contacting surface element pairs was collected and distributed using CM FORTRAN intrinsic functions PACK and UNPACK. The distribution of pinball data to every processor allowed the search for interacting pairs to proceed in parallel. 


\section{ACKNOWLEDGEMENTS}

Computations were performed on the $16 \mathrm{~K}$ processor Connection Machine at the National Center For Supercomputing Applications, University of Illinois, ChampaignUrbana and the Army High-Performance Computing Research Center, Minnesota Supercomputing Center, Minneapolis. The support of the Department of Educational Programs, and Computing and Telecommunications Division at Argonne National Laboratory is gratefully acknowledged.

\section{REFERENCES}

1. Belytschko, T., Chiapetta, R. L., and Bartell, H. (1976) "Efficient Large Scale Nonlinear Transient Analysis by Finite Elements," International Journal For Numerical Methods In Engineering. 10, 579-596.

2. Flanagan, D. P. and Belytschko, T. (1981) “A Uniform Strain Hexahedron and Quadrilateral with Orthogonal Hourglass Control," International Journal For Numerical Methods In Engineering. 17, 679-706.

3. Belytschko, T. (1983) "An Overview of Semidiscretization and Time Integration Procedures," Computational Methods for Transient Analysis, eds. T. Belytschko and T. J. R. Hughes, 1-65. North Holland, Amsterdam.

4. Belytschko, T., Plaskacz, E. J., Kennedy, J. M., and Greenwell, D. L. (1990) "Finite Element Analysis on the CONNECTION Machine," Computer Methods In Applied Mechanics and Engineering. 81(2), 229-254. 
5. Plaskacz, E. J. and Belytschko, T., "Measurement and Exploitation of Mesh Structure in a SIMD Environment," Proceedings of the 1990 ASME International Computers In Engineering Conference and Exposition, II. New York: American Society of Mechanical Engineers, pp. 559-566, August 1990.

6. Belytschko, T., Plaskacz, E. J. (1992) "SIMD Implementation of a Nonlinear Transient Shell Program with Partially Structured Meshes," International Journal For Numerical Methods In Engineering. 33, 997-1026.

7. Belytschko, T. and Law, S. E. (1985) “An Assembled Surface Normal Algorithm for Interior Node Removal in Three-dimensional Finite Element Meshes," Computers and Structures : 25, 95-104.

8. Belytschko, T. and Lin, J. I. (1987) "A Three-dimensional Impact-Penetration Algorithm with Erosion," Engineering with Computers : 25, 95-104.

9. Belytschko, T. and Neal, M. O. (1991) "Contact-Impact by the Pinball Algorithm with Penalty and Lagrangian Methods," International Journal For Numerical Methods In Engineering. 31, 547-572. 
List of Figures

Figure 1: Von Neumann and SIMD Data Structures

Figure 2: Four Stage Internal Force Exchange

Figure 3: NEWS - Router Hybrid Interprocessor Communication

Figure 4: Pinballs Injected Into Elements With Non-zero Surface Normals

Figure 5: Parallel Computation of Nodal Surface Normals

Figure 6: Parallel Assembled Surface Normal Algorithm and Pinball Injection

Figure 7: Two-Body Contact-Impact Simulation: Mapping of Finite Elements to Connection Machine Processors 


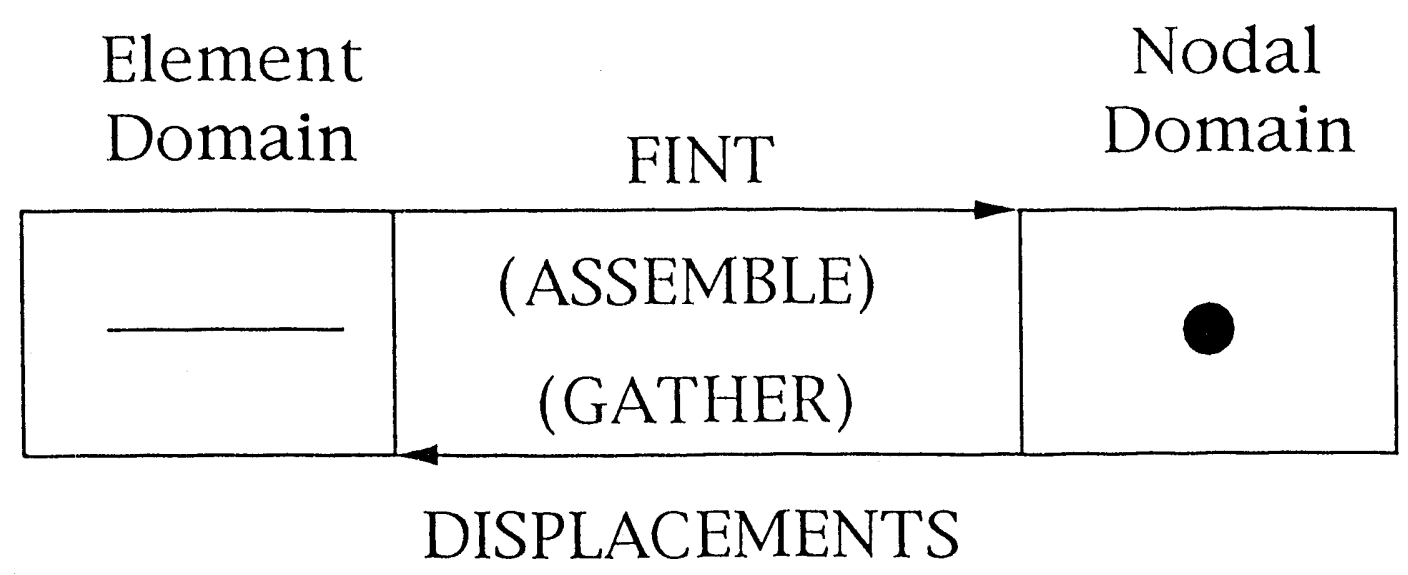

Figure 1a Von Neumann Data Structure

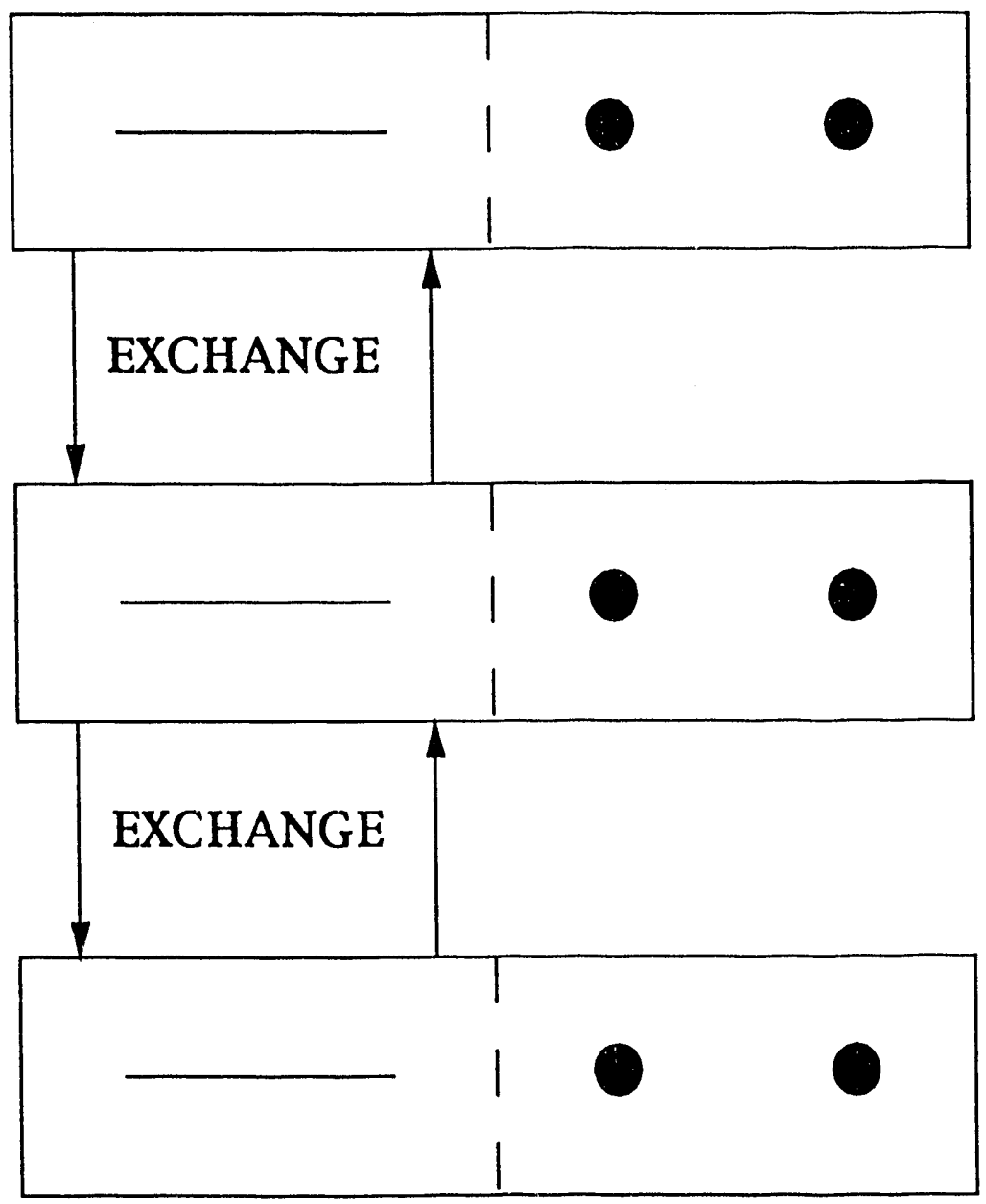

Figure 1b SIMD Data Structure

Figure 1: Von Ncumann and SIMD Data Structures 


\begin{tabular}{|l|l|l|}
\hline 7 & -8 & -9 \\
\hline 4 & -5 & -6 \\
\hline $1-2$ & -3 \\
\hline
\end{tabular}

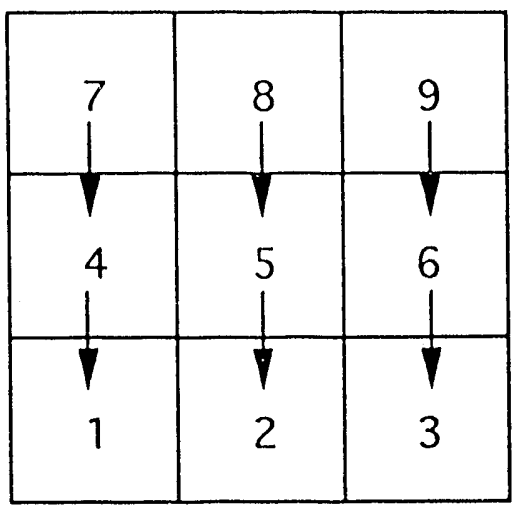

Stage 2:

Stage 4:

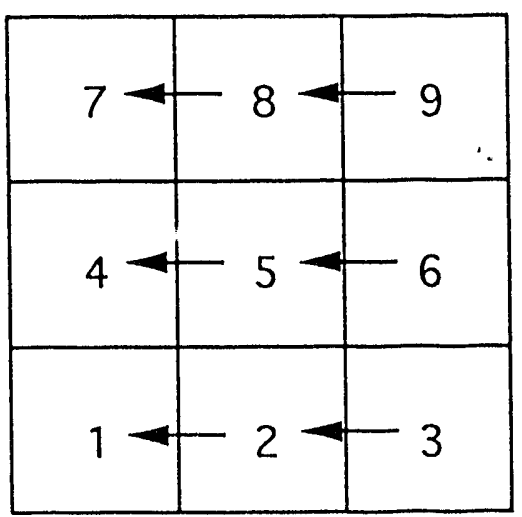

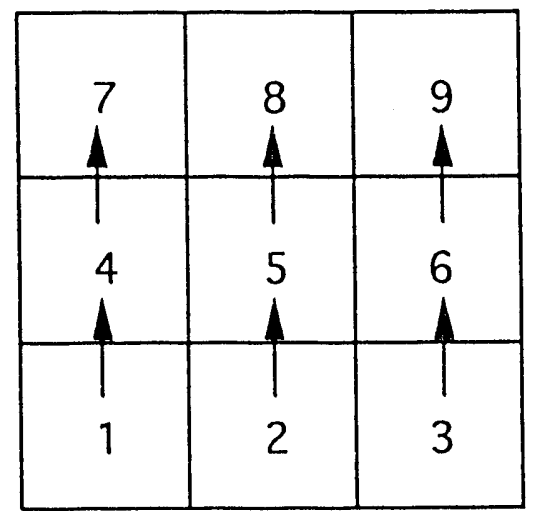

Figure 2: Four Stage Exchange of Internal Forces 


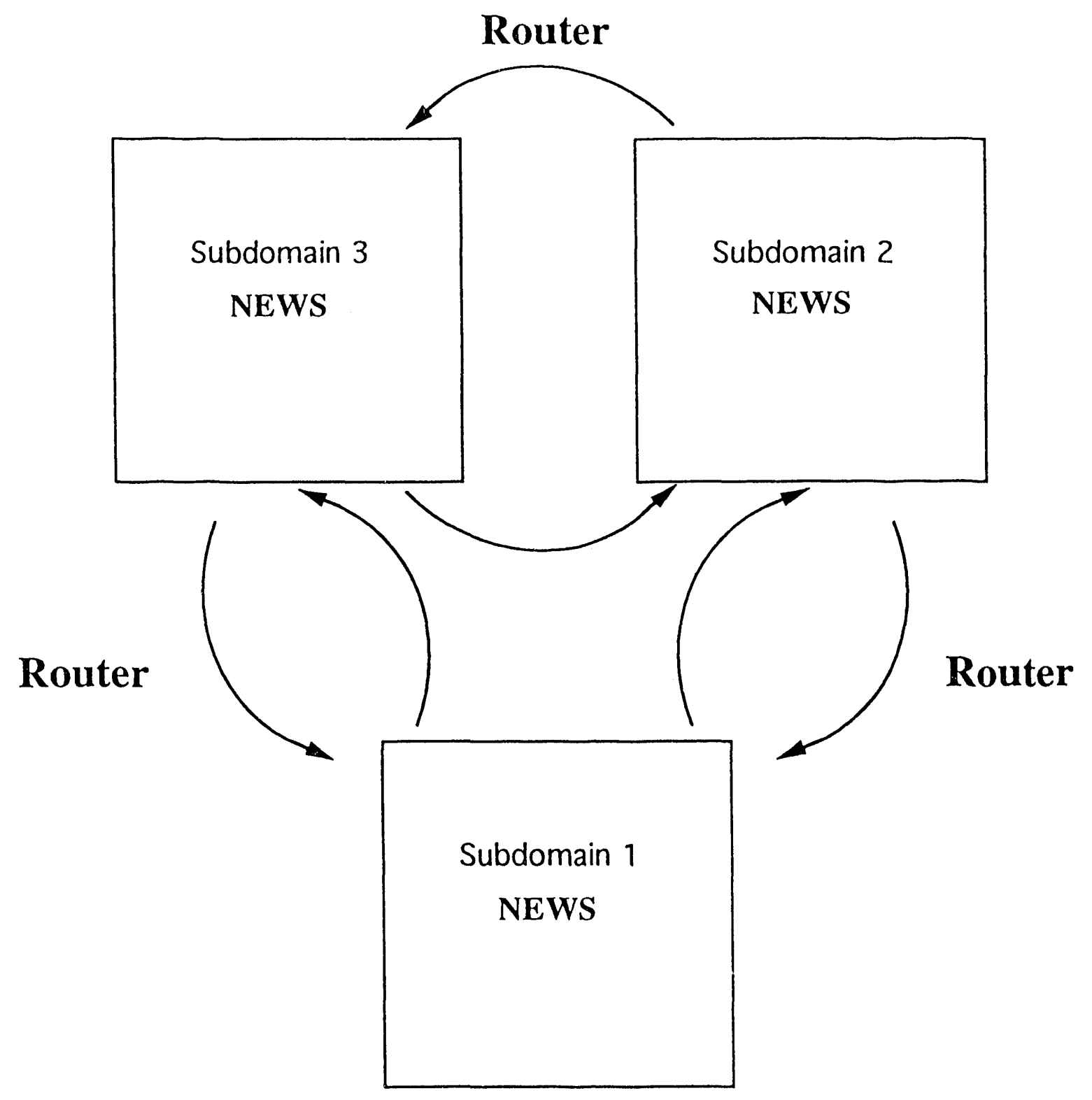

Figure 3: NEWS - Router Hybrid Interprocessor Communication 


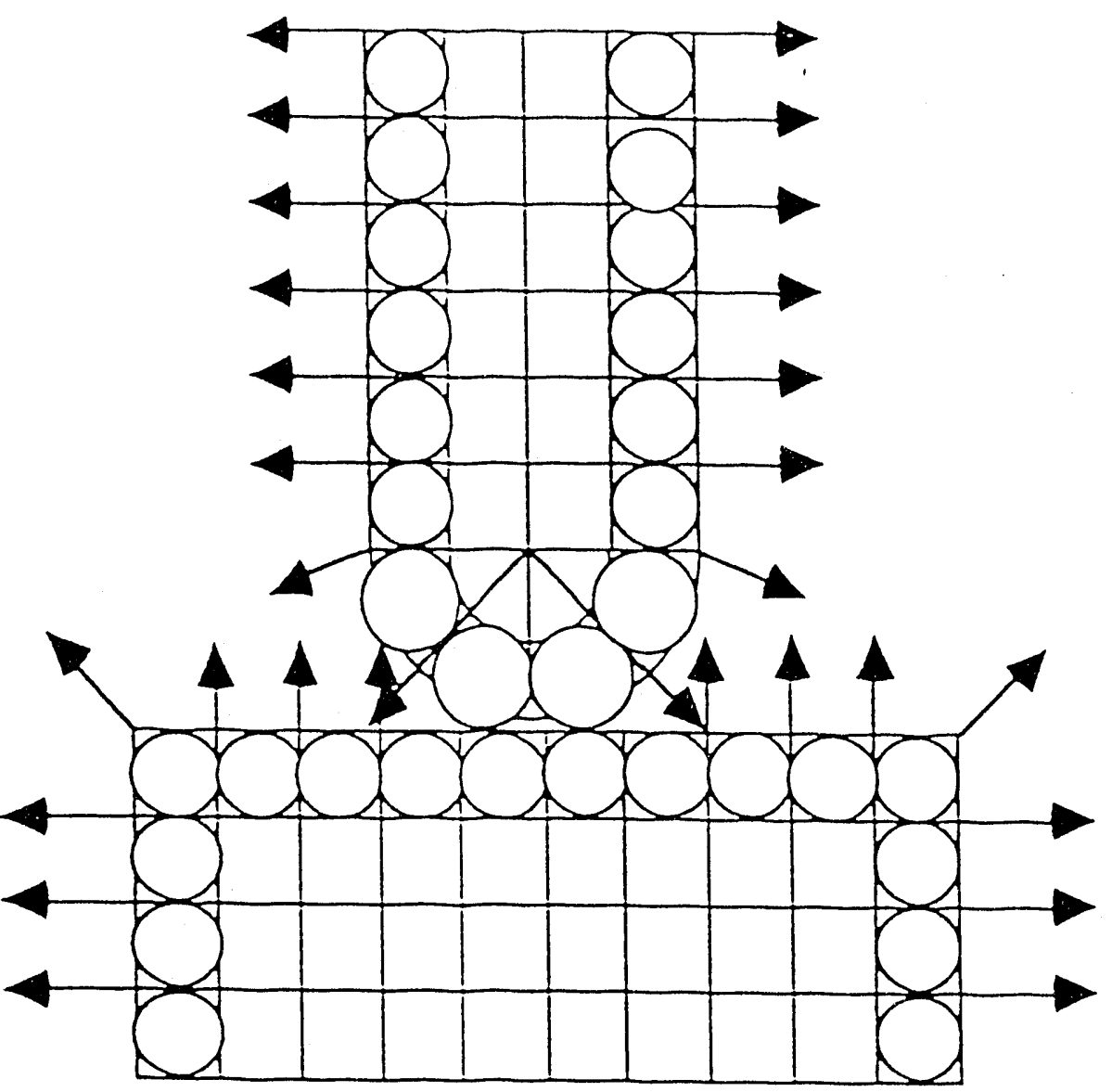

Figure 4: Pinballs Injected Into Elements With Non-zero Surface Normals 


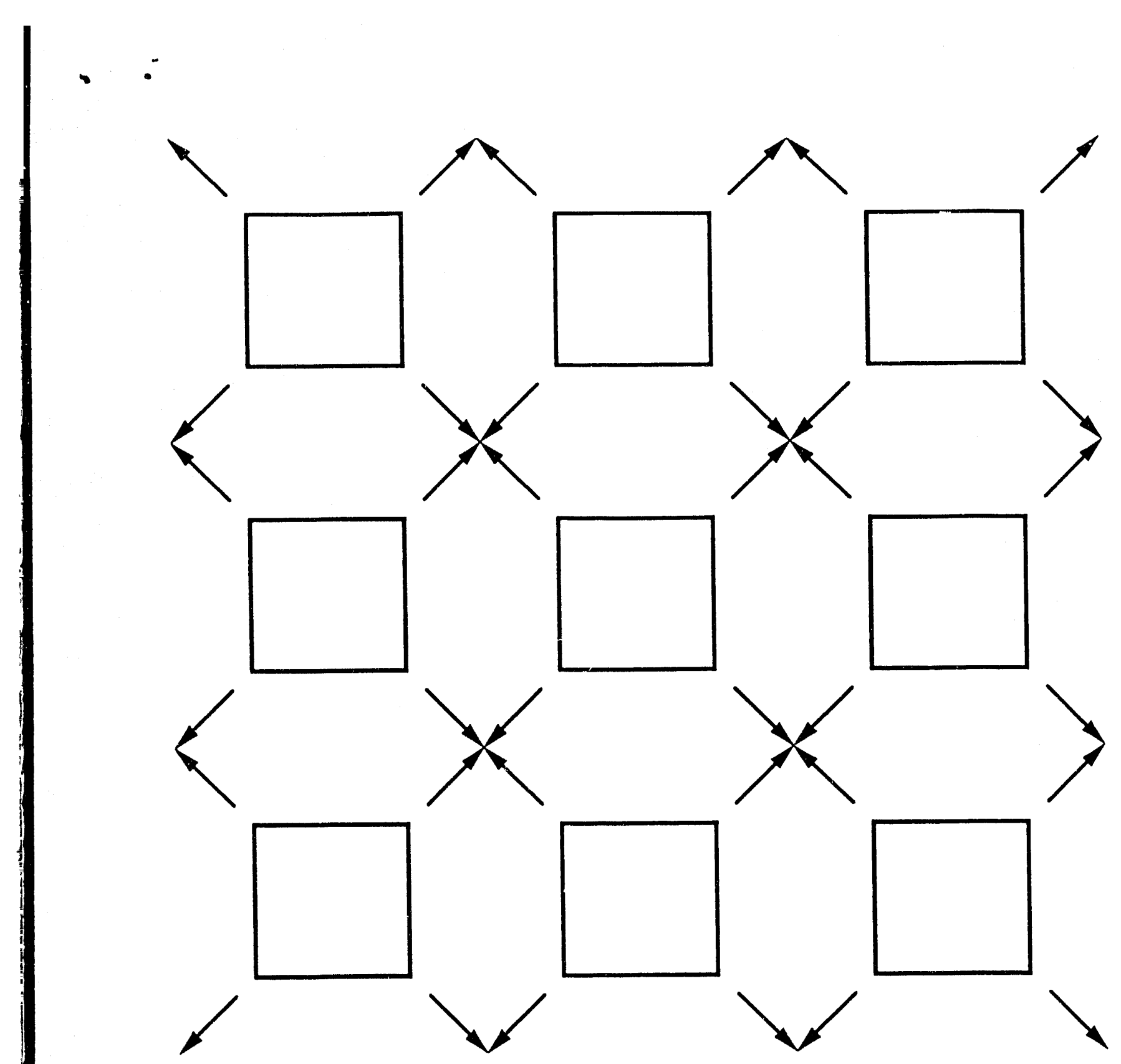

Figure 5: Parallel Computation of Element Surface Normals 

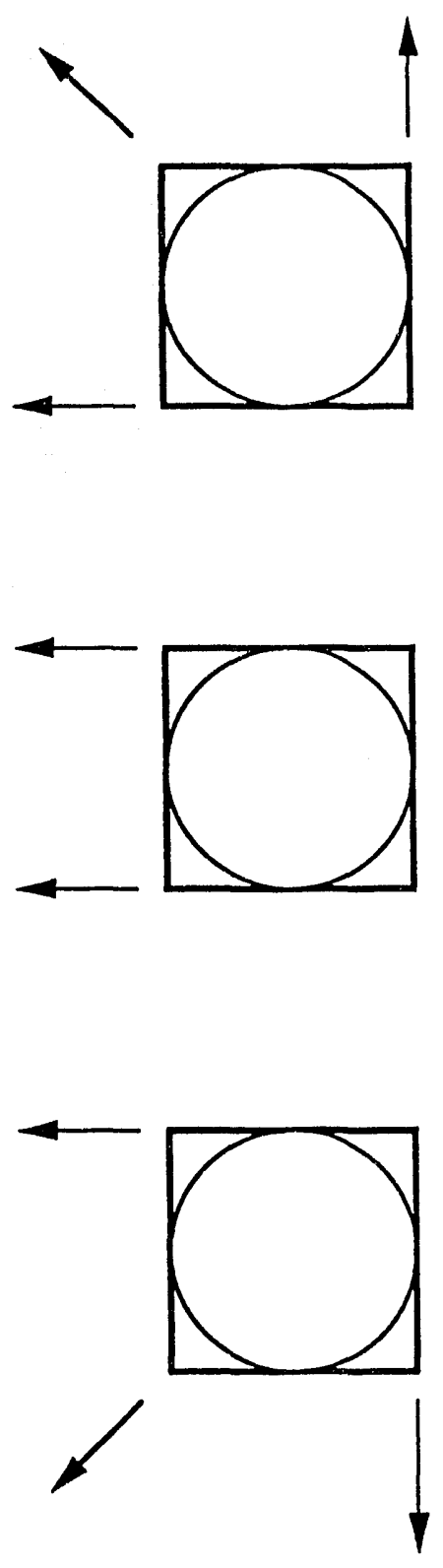
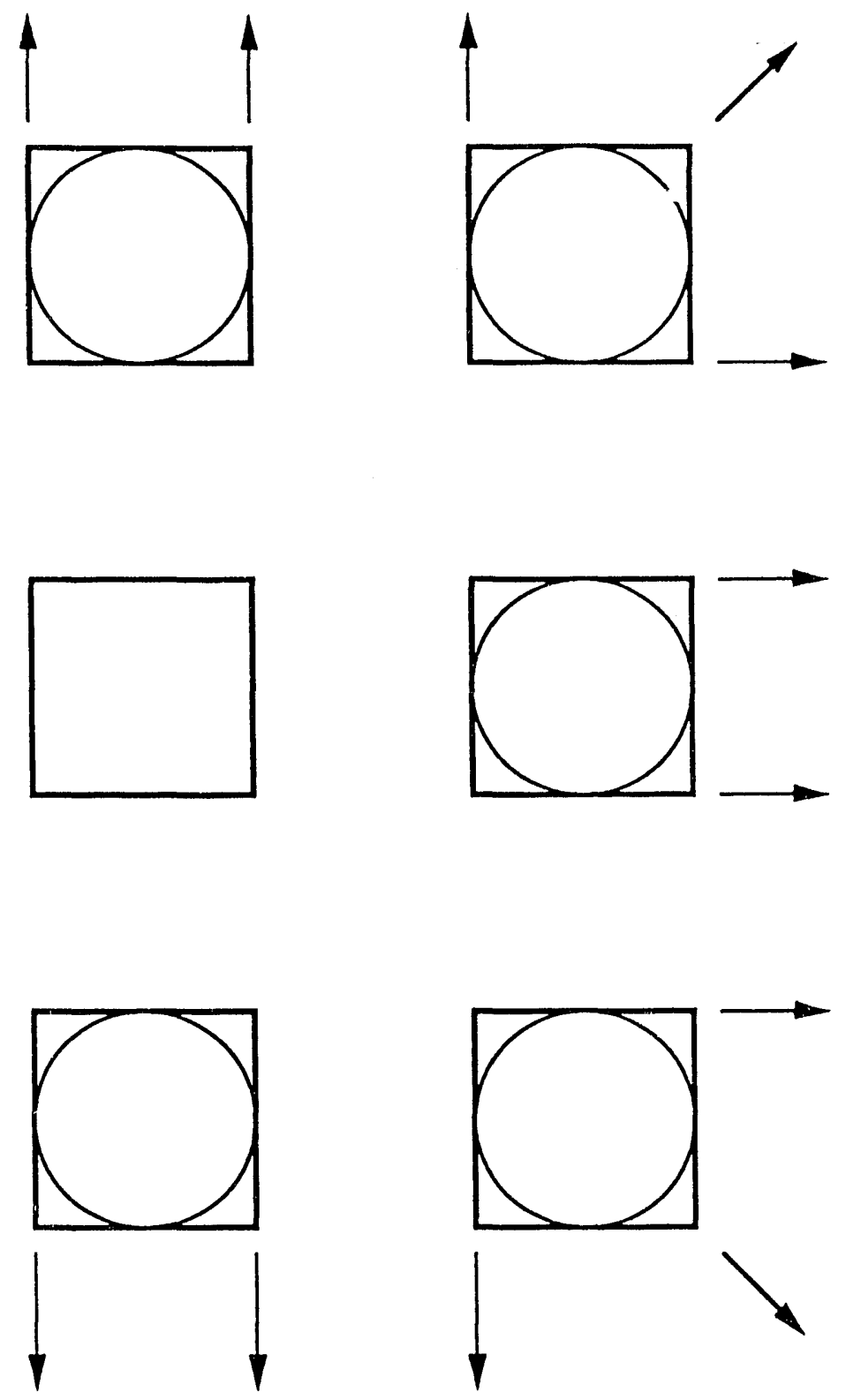
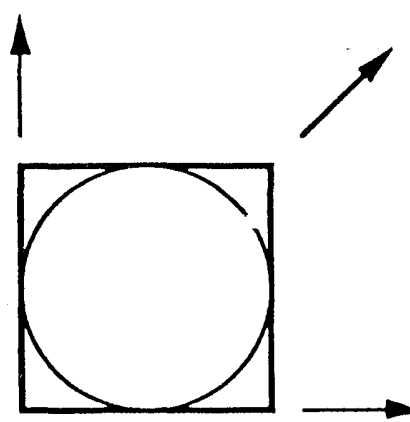

$\longrightarrow$
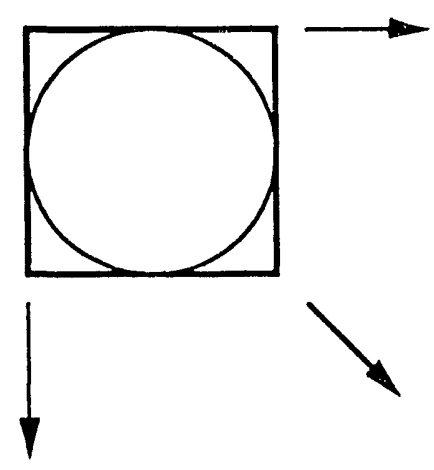

Figure 6: Parallel Assembled Surface Normal Algorithm and Pinnball Injection 


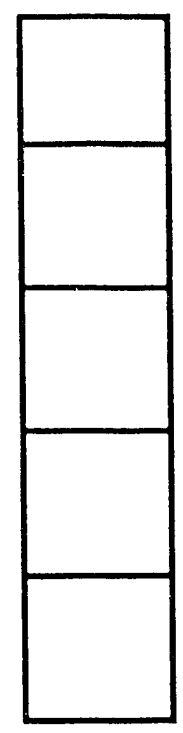

Slave

(Penetrant)

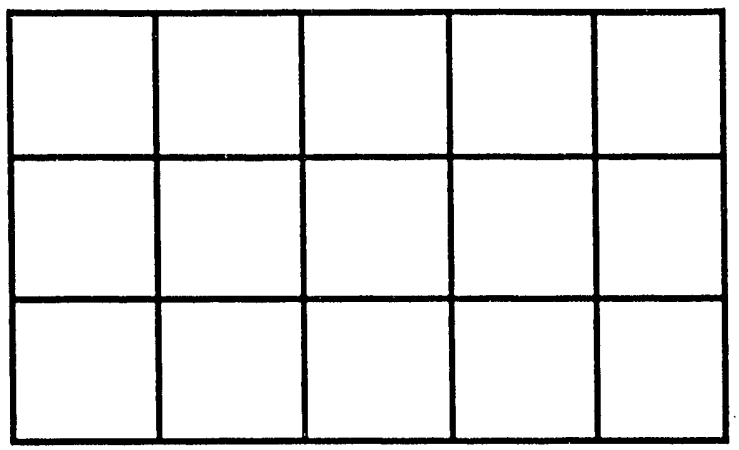

Master

(Target)

Slave

(Penetrant)

Master

(Target)

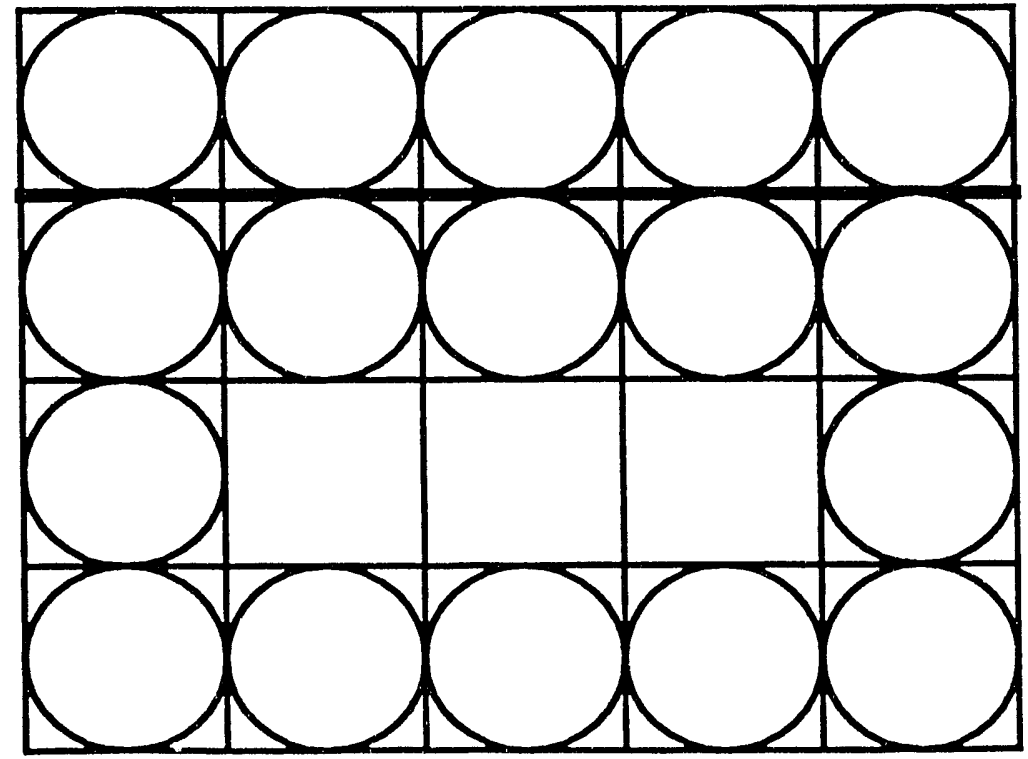

Figure 7: Two-Body Contact-Impact Simulation: Mapping of

Finite Elements to Connection Machine Processors 


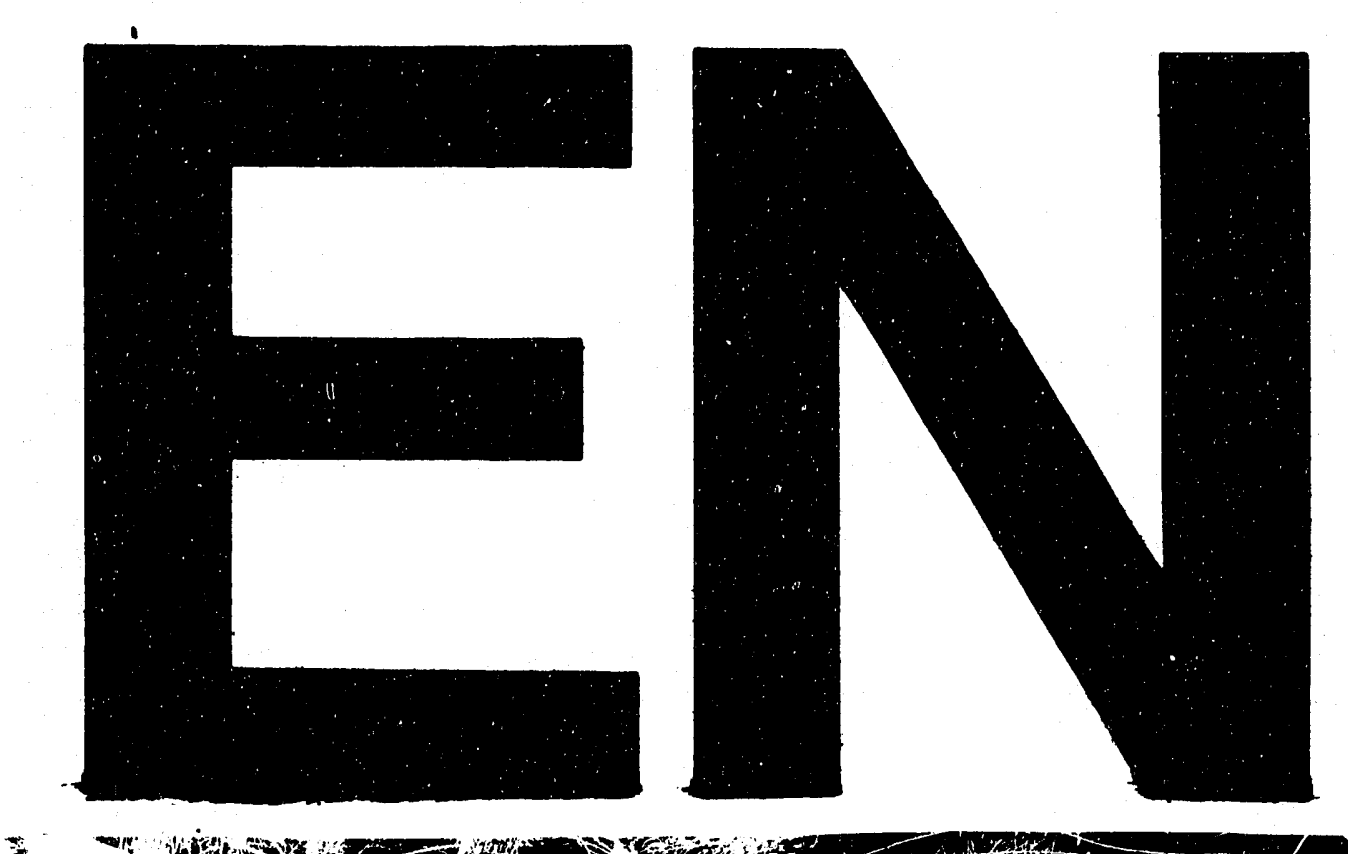

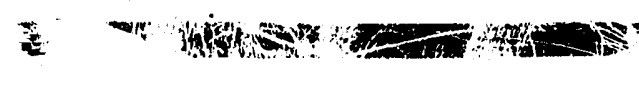
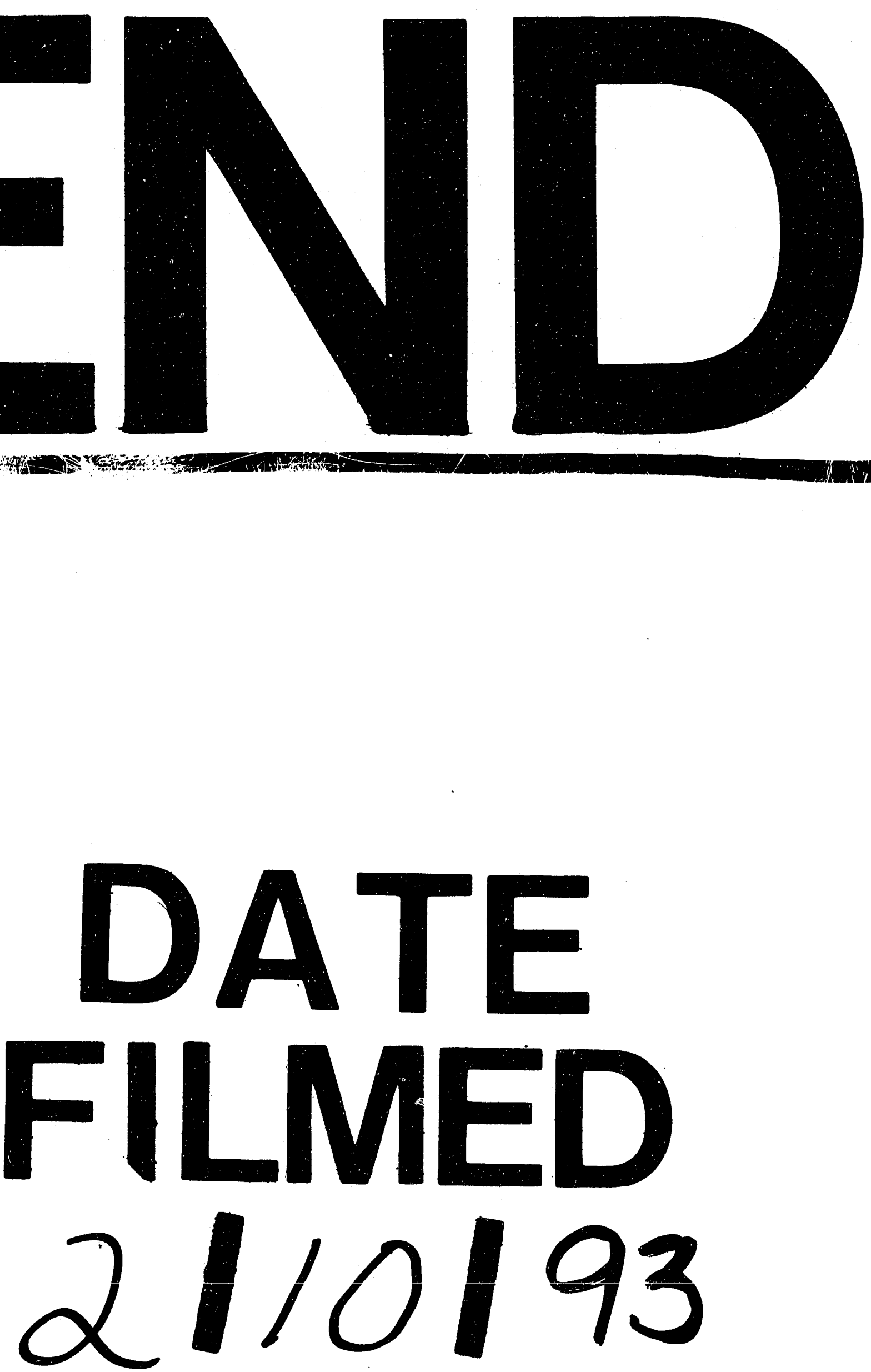

言 


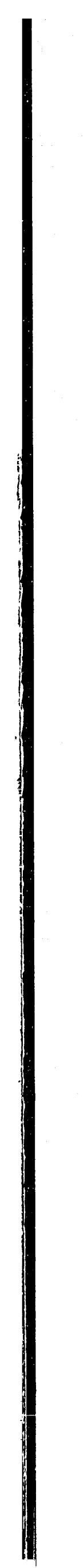

